\title{
SPECTROSCOPY OF THREE SBS GALAXIES
}

\author{
M.V. GYULZADIAN
}

Byurakan Astrophysical Observatory, 378433 Byurakan, Armenia

Three galaxies from Second Byurakan Sky Survey have been studied. The spectra of these galaxies were obtained at 6-m telescope of the Special Astrophysical Observatory of the Russian Academy of Sciences. For these objects line widths and emission line ratios were measured and ionization mechanism and chemical abundances were determined.

The FWHM for the all galaxies is in order of FWHM for narrow emission line galaxies. The excitation mechanism for them is photoionization.

Observed [SII] $/ \mathrm{H} \alpha$ ratios are rather large in comparison to more conventional HII regions.

On the base of FWHM of emission lines, observed in the spectra of SBS $1122+610$ and SBS $1139+601$, these objects were placed on the diagrams of Baldwin et al. (1981) and Veilleux \& Osterbrock (1987), and it was concluded that these galaxies are NELGs. SBS $1133+597$ is HII galaxy.

\section{References}

Baldwin, J.A., Philips, M.M., Terlevich, R. 1981, PASP 93, 5

Veilleux, S., Osterbrock, D. 1987, ApJS 63, 295 